\title{
Philosophiques
}

\section{L'herméneutique métaphysique entre metaphysica generalis et metaphysica specialis}

\section{Guillaume St-Laurent}

Volume 41, numéro 2, automne 2014

URI : https://id.erudit.org/iderudit/1027226ar

DOI : https://doi.org/10.7202/1027226ar

Aller au sommaire du numéro

Éditeur(s)

Société de philosophie du Québec

ISSN

0316-2923 (imprimé)

1492-1391 (numérique)

Découvrir la revue

Citer ce document

St-Laurent, G. (2014). L'herméneutique métaphysique entre metaphysica generalis et metaphysica specialis. Philosophiques, 41(2), 371-378.

https://doi.org/10.7202/1027226ar d'utilisation que vous pouvez consulter en ligne.

https://apropos.erudit.org/fr/usagers/politique-dutilisation/ 


\title{
L'herméneutique métaphysique entre metaphysica generalis et metaphysica specialis
}

\author{
GUILLAUME ST-LAURENT \\ Université de Montréal \\ guil.stlaurent@gmail.com
}

Dans un style toujours aussi éloquent et rigoureux, le dernier ouvrage de Jean Grondin propose d' « esquisser quelques-unes des idées directrices d'une herméneutique métaphysique ${ }^{1}$ ", de manière à montrer l'actualité du platonisme, c'est-à-dire de la métaphysique traditionnelle des Idées et du souverain Bien, dans l'horizon herméneutique de la philosophie contemporaine. En ce sens, nous interprétons le geste général de cette «herméneutique métaphysique " comme une tentative de synthétiser (i) les perspectives ouvertes par l'herméneutique post-heideggérienne - notamment chez Hans-Georg Gadamer et Paul Ricœur - touchant la portée ontologique de la compréhension humaine, d'une part, ainsi que (ii) l'hypothèse inaugurale ou le "grand pari» de la métaphysique classique voulant qu'il faille reconnaitre dans l'ordre intelligible du monde la manifestation d'un principe transcendant ou divin, d'autre part. Cet audacieux effort d'unification, soutenu par un argumentaire mûri et nuancé, doit permettre à la fois de réhabiliter et de renouveler le projet essentiel de la métaphysique: «Tout platonicien pressent, parie, que la réalité immédiatement visible, sensible, temporelle, n'est peutêtre pas la seule. Son idée ou son herméneutique de fond est que le sensible laisse lui-même transparaître, par sa beauté (eidos), une réalité digne d'être appelée "méta-physique"."

Ainsi, le premier aspect de cette herméneutique métaphysique que nous aimerions souligner consiste en sa critique du nominalisme moderne au nom d'un certain réalisme herméneutique (ou de ce que notre auteur présenta ailleurs comme "la thèse de l'herméneutique sur l'être $\left.{ }^{3} »\right)$, attribuable aussi bien à l' "herméneutique philosophique » de Gadamer qu'à la «phénoménologie herméneutique» de Ricœur. Alors que le nominalisme ambiant stipule que "[...] tout le monde du sens se résume à une production de la pensée qui projette ses concepts généraux sur le monde, lequel se compose de masses physiques individuelles, intrinsèquement dépourvue de sens ${ }^{4}$ », le réalisme herméneutique entend montrer que notre compréhension s'emploie d'abord et avant tout à expliciter le «sens des choses elles-mêmes». Loin de se réduire à de simples «noms » (nomina) ou constructions sociolinguistiques,

1. Jean Grondin, 20I3, p. VI.

2. Ibid., p. I 29.

3. Jean Grondin, «La thèse de l'herméneutique sur l'être ", 2006.

4. Jean Grondin, 20I3, p. I70.

PHILOSOPHIQUES 41/2 - Automne 2014, p. 371-378 
les «idées » renverraient plus fondamentalement à l'intelligibilité intrinsèque des phénomènes ou à la «dicibilité de principe ${ }^{5}$ » de l'expérience. De même, à l'encontre des herméneutiques antiréalistes ou post-modernes de Jacques Derrida, Michel Foucault, Richard Rorty ou Gianni Vattimo, qui rejettent plus ou moins nettement la distinction entre l'ordre de nos interprétations et celui de la réalité ( «[...] l'être se réduit aux interprétations que l'on en donne $\left.^{6} »\right)$, notre horizon langagier et historique nous ouvrirait un accès fini à l'être comme tel, soit au monde en tant que réalité unique et transcendante. Notre finitude nous confinerait à la "vérité-adéquation» entendue comme une visée $(a d)$ transitive et toujours inachevée de l'équation (aequatio) ou de l'équité avec l'être-vrai, la pleine identité de la connaissance et de l'être étant ex hypothesi réservée à Dieu, mais l'intelligence humaine demeurerait la capacité de percevoir et penser l'intelligence des choses elles-mêmes7. C'est dire que l'herméneutique devrait être comprise comme une phénoménologie - visant à "faire voir les phénomènes ${ }^{8}$ " dans leurs articulations autochtones - et la phénoménologie comme une herméneutique, ou encore que le tournant ontologique de l'herméneutique coïncide rigoureusement avec le tournant herméneutique de la phénoménologie.

Cela dit, ce réalisme herméneutique mérite-t-il à lui seul le qualificatif de "métaphysique " ? Vraisemblablement, le terme conviendra si le concept de métaphysique est entendu de manière générale comme la tentative de "comprendre l'être à partir de ses raisons", ou comme "le projet d'une compréhension du monde, à vocation universelle, qui s'interroge sur l'être et le pourquoi des choses ${ }^{9}$ " - instaurant dès lors une quadruple équivalence entre philosophie, phénoménologie, herméneutique et métaphysique. Comme le souligne notre auteur dans la section de l'épilogue intitulée "Deux témoignages révélateurs », cette équation se trouvait déjà chez Gadamer lui-même, qui était bien conscient que sa thèse célèbre énonçant que "l'être qui peut être compris est langage », laquelle signifiait en substance que notre compréhension dépend et se règle sur la «langue des choses » (die Sprache der Dinge $)^{10}$, reconduit la pensée dans l'horizon de la métaphysique classique: «[...] [L]'herméneutique ne nous conduit pas au-delà de la métaphysique, bien au contraire, elle nous y replonge ${ }^{11}$.» C'est d'ailleurs l'une des grandes réussites de l'Introduction à la métaphysique (2004) et Du sens des choses que de faire ressortir cette évidence, occultée par le nominalisme

5. Paul Ricœur (I975), "Phénoménologie et herméneutique: en venant de Husserl...», I986, p. 62.

6. Jean Grondin, «La thèse de l'herméneutique sur l'être ", 2006, p. 47I.

7. Jean Grondin, 2013, p. 93-100.

8. Ibid., p. I20.

9. Jean Grondin, 2004, p. I3.

10. Hans-Georg Gadamer, I960, "La nature de la "res" et le langage des choses ", trad. I99I.

11. Jean Grondin, 2013, p. I45. 
et le subjectivisme modernes, de la coappartenance transcendantale de la pensée et de l'être, puisqu'il est impossible pour la raison humaine de ne pas présupposer une conception de l'être véritable des choses et, partant, de critiquer la métaphysique sans la présupposer et l'accomplir ${ }^{12}$.

Toutefois, l'idée d'une "herméneutique métaphysique » serait dans ces conditions elle-même équivalente à celle d'une phénoménologie herméneutique ou d'une herméneutique philosophique, ce qui risquerait de nous faire perdre de vue l'originalité de la première. De fait, notre auteur souligne à la toute dernière page de l'ouvrage que l'intelligence ontologique du langage chez Gadamer prépare sur le plan de la metaphysis generalis ou de l'ontologie, mais sans s'y aventurer, "la problématique d'une metaphysis specialis" qui mériterait d'être développée plus avant ${ }^{13}$. Cela indique que le réalisme herméneutique n'épuise pas la position de notre auteur et que celle-ci peut aussi être dite "métaphysique " en un sens plus précis, c'est-à-dire non seulement dans la mesure où elle met en œuvre (a) une ontologie portant sur les propriétés ou les déterminations les plus générales de l'être, reconnaissant que le monde est pour nous d'emblée sensé et intelligible, mais également parce qu'elle se prononce (b) au plan de la métaphysique spéculative sur le "principe transcendant de ce qui est» ou sur le «sens ultime» des choses.

En effet, le professeur Grondin nous exhorte à reconnaître dans la sixième leçon de l'ouvrage ( «Du sens métaphysique »), par-delà la modestie métaphysique du réalisme herméneutique, que l'ordre intelligible des choses laisse "pressentir» une intelligence ordonnatrice et transcendante, un Premier principe ou un souverain Bien responsable de cet ordre: "La meilleure métaphysique, celle qui prend au sérieux l'intelligence des choses, est celle qui pressent que l'immanence laisse deviner quelque transcendance et que la transcendance n'en est pas une si elle n'investit pas l'immanence ${ }^{14}$. "Cet argument est de facture classique, et son ressort essentiel n'est autre que le dépassement du nominalisme moderne dans la reconnaissance de l'harmonie intelligible du monde (argument $e$ sensu rerum) ainsi que de notre insigne capacité d'en comprendre quelque chose (argument e intelligentia rerum). Dès lors, le réalisme herméneutique se meut et se radicalise en une authentique "herméneutique métaphysique ", selon laquelle le "sens des choses" n'est plus seulement transcendant du point de vue phénoménologique, sur le mode de la "transcendance dans l'immanence» (ou de l'en-soi-pour-nous), mais bien métaphysiquement transcendant, c'est-à-dire subsistant indépendamment de notre existence incarnée et située. Autrement dit, cette thèse de métaphysique spéciale donne un tour proprement méta-physique - au sens suprasensible ou spéculatif du terme - aux réflexions transcendantales et

12. Ibid., p. 6; Jean Grondin, 2004, p. I3.

13. Ibid., p. I70. Pour cette distinction entre métaphysique générale/spéciale, voir: Ibid., p. I 2.

14. Ibid., p. I26. 
phénoménologiques sur les conditions de la compréhension humaine, accomplissant le passage d'un réalisme sans fondement ontothéologique, qui reconnaissait déjà aux idées une positivité ontologique irrécusable, à une forme générale de platonisme leur accordant une existence séparable de la nôtre, "une plus haute réalité ${ }^{15}$ ». Le sens de l'être s'ancrerait lui-même dans un sens méta-physique qui le fonde et permet d'en rendre raison (logon didonai); la raison humaine se fonderait sur l'adéquation plus fondamentale de l'intellect divin et de l'être.

Or, bien que présenté comme le prolongement naturel et raisonnable du réalisme herméneutique de ses prédécesseurs, notre auteur n'est pas sans savoir - il le sait mieux que quiconque — que Gadamer aurait refusé un tel parachèvement théologique de son herméneutique philosophique. En effet, une herméneutique qui ferait dépendre son orientation "réaliste» d'une thèse spéculative de ce type, en recourant à une intelligence transcendante pour rendre compte de la correspondance transcendantale de la pensée et de l'être, escamoterait selon lui le défi central de la philosophie contemporaine:

Il est certes vrai que la philosophie ne peut plus aujourd'hui se servir d'une pareille explication théologique, ni en répéter les formes sécularisées comme les représente l'idéalisme spéculatif avec sa conciliation dialectique du fini et de l'infini. Mais, de son côté, elle n'a pas le droit de se fermer à la vérité de cette correspondance [de la pensée et de l'être]: en ce sens, la tâche de la métaphysique apparaît assurément comme ne pouvant plus être métaphysique, c'est-à-dire comme recourant à un intellect infini. La question qui se pose est alors celle-ci: Y a-t-il des possibilités finies qui puissent rendre compte de cette correspondance? Y a-t-il une justification de cette correspondance qui ne recoure pas à un esprit divin infini, mais qui puisse toutefois rendre compte de cette correspondance infinie de l'âme et de l'être? ${ }^{16}$

La tâche de la métaphysique - ou, indifféremment, de la phénoménologique, de l'herméneutique et de la philosophie - consisterait donc désormais à élucider de manière immanente le phénomène herméneutique, à justifier en termes de "possibilités finies» notre capacité de comprendre le sens des choses elles-mêmes, plutôt que par un quelconque expédient théologique relevant de la métaphysique spéciale. Mais que pense vraiment le professeur Grondin de cette réticence chez Gadamer? Ce dernier aurait-il succombé au «faux principe du hasard ${ }^{17}$ » ou à «quelque bien-pensance antimétaphysique ", à défaut de suivre la voie royale tracée par la métaphysique

15. Ibid., p. I32.

16. Hans-Georg Gadamer, I960, "La nature de la "res" et le langage des choses ", trad. I99I, p. I 29.

17. "L'herméneutique métaphysique qui ne se laisse pas impressionner par la faveur dont jouit le faux principe $d u$ hasard - faux principe car il n'explique rien et si, d'aventure, il vient expliquer quelque chose, il n'est pas le hasard - redécouvre que ce n'est pas un hasard si l'intelligence des choses a conduit à peu près tous les philosophes à admettre l'évidence d'un premier principe responsable de cette intelligence» (Jean Grondin, 2013, p. I33). 
classique? Le «chemin du langage » emprunté par Gadamer afin de répondre à ce défi était-il en définitive un cul-de-sac métaphysique, vu qu'il est impossible de «soutenir l'idée d'une adéquation fondamentale entre l'être et le langage sans arrière-plan théologique ${ }^{18} »$ ?

Le défi susmentionné présuppose vraisemblablement une distinction épistémologique forte entre metaphysica generalis et metaphysica specialis, ou encore entre ontologie et ontothéologie, ayant pour effet de refouler la seconde hors de l'espace public de la raison philosophique vers les domaines de l'art, de la quête spirituelle et de la foi. En ce sens, compte tenu du fait que la métaphysique dans son ensemble ne saurait constituer une science apodictique (empirique ou déductive), mais bien plutôt une quête vigilante, faillible et autocritique des principes ou des fondements de l'être ${ }^{19}$, la question déterminante nous paraît être la suivante: comment la métaphysique peut-elle légitimement aspirer à dépasser le plan des opinions, qui admet déjà la vraisemblance et la «justesse ${ }^{20}$ », vers celui des convictions, c'est-àdire des certitudes subjectives fondées sur des raisons jugées suffisantes? Dans un premier temps, la réponse de la plupart des philosophies transcendantales et phénoménologiques consiste à soutenir que la métaphysique générale peut préserver sa vocation a priori (fondamentale et universelle) en infléchissant son effort vers la "finitude » de la rationalité humaine, c'est-àdire vers ses conditions, possibilités et limites. Autrement dit, l'ontologie pourrait encore commander la conviction de chacun en explicitant les conditions de possibilité/intelligibilité de la compréhension ou ses "arrièreplans $^{21} »$ (corporéité, historicité, linguisticité, etc.), quand bien même cette première implique un travail à jamais inachevé de déconstruction et d'argumentation. Mais qu'en est-il de la métaphysique spéciale? L'ontothéologie peut-elle encore prétendre dépasser le niveau des seules assurances sans pour cela trouver appui dans quelque forme de révélation personnelle ou officielle, c'est-à-dire sans consommer le passage de la « raison publique » à la «raison privée ${ }^{22}$ ?

18. Cf. Jean Grondin, «La thèse de l'herméneutique sur l'être », 2006, p. 48I.

19. Cf. Jean Grondin, 20I3, p. 2I-32.

20. Ibid, p. 78 .

21. Cf. Charles Taylor (I987), "Overcoming Epistemology", I995, p. 8-I3.

22. Alors que cette distinction connaît un usage courant en philosophie politique, nous pensons que le concept de «raison privée » pourrait aussi désigner un vaste domaine de questionnement où la frontière entre ce qui est «accessible » (c.-à-d. communicable et valable pour tous) et ce qui est «inaccessible» (c.-à-d. ne pouvant être admis que par un sacrificium intellectus) à la raison s'avère à la fois relative et mobile. En nous inspirant du modèle "ad hominem" développé par Charles Taylor, la raison privée consisterait ainsi en un mode de rationalité où certaines raisons pourraient être accessibles à partir d'un point de vue ' $B$ ' (en vertu d'un itinéraire ou d'expériences personnelles) tout en étant inaccessibles d'un point de vue ' $A$ ' (non pas de manière a priori insurmontable, mais à divers degrés) et sans pour autant cesser d'être des «raisons» : "There are good reasons; it is just that they are only available from standpoint B." 
Il est vrai que Kant lui-même avait déjà transgressé dans sa «métaphysique des mœurs » les limites de l'expérience possible en s'autorisant à penser le souverain Bien à titre de "postulat de la raison pratique ${ }^{23}$. Toutefois, nous pourrions objecter que celui-ci ne manqua pas de situer ce geste dans le registre de l'espérance et de la «foi morale» plutôt que dans celui du savoir ${ }^{24}$, d'une part, et que la conscience historique signifie d'abord et avant tout l'historicisation (ou la relativisation historique) de ce type de "théologie transcendantale", d'autre part: à peu près rien dans l'ordre des vérités dernières n'est évident à partir de tous les points de vue ou de toutes les traditions historiques. De fait, il semble que notre situation herméneutique soit telle que les arguments spéculatifs (théologiques ou "athéologiques») qui partent de la condition humaine ou de réalités accessibles à tous - par exemple: la liberté humaine, l'existence du mal, le désir d'immortalité et de consolation, l'ordre et la beauté du monde, etc. - ne parviennent en définitive à persuader que ceux qui en auront déjà accepté les conclusions. En ce sens, nous pourrions invoquer avec Charles Taylor un certain primat de l'expérience morale et spirituelle dans le domaine de la métaphysique spéciale, sans forcément demeurer captif des alternatives des Lumières: la pluralité indéniable des traditions et la sécularisation de l'Occident chrétien attestent du fait que les arguments spéculatifs ne parviennent guère à produire l'assentiment chez un grand nombre de penseurs honnêtes et lucides, de sorte que seule l'expérience personnelle semble pouvoir justifier les convictions en cette auguste matière ${ }^{25}$.

Par ailleurs, nous voyons deux autres motifs pour refuser de rendre compte de cette " $[. .$.$] intelligence des choses que nous ne cessons jamais de$ comprendre, de présupposer et d'admirer ${ }^{26}$ » par une hypothèse explicative relevant de la théologie naturelle. Tout d'abord, comme le souligne à bon droit André Comte-Sponville, cette démarche soulève le problème d'une "explication incompréhensible» : elle consiste à vouloir expliquer ( «rendre clair») quelque chose que nous comprenons très peu par quelque chose que nous comprenons encore moins ${ }^{27}$. Notons que le principe épistémologique sur lequel se fonde cet argument n'a rien de positiviste ni de scientiste, puisqu'il ne s'agit pas de promouvoir une «science sans métaphysique » ou

(Charles Taylor, «A Philosopher's Postscript: Engaging the Citadel of Secular Reason ", 2005, p. 34I).

23. Jean Grondin, 20I3, p. I I.

24. La foi morale n'est pas chez Kant un savoir, mais bien un type de certitude s'appuyant sur les fondements subjectifs de ma «disposition morale» (moralische Gesinnung), de sorte que "[...] je ne dois même pas dire: Il est moralement certain qu'il y a un Dieu, etc., mais: Je suis moralement certain, etc. " (Emmanuel Kant, $198 \mathrm{I}-\mathrm{I} 987$, Critique de la raison pure, trad. I980, I, A 829/B 857, p. I382).

25. Cf. Charles Taylor, «Reply and re-articulation», I994, p. 226-228.

26. Jean Grondin, 20I3, p. I33.

27. André Comte-Sponville, 2006, p. I IO-I I 8. 
une "science comme métaphysique ${ }^{28}$ ". Plutôt, étant entendu que nous ne pouvons comprendre que «bien peu de choses » au souverain Bien ${ }^{29}$, l'explication théologique est vaine parce que nous devrions mieux comprendre ce par quoi nous expliquons (l'explanans) que ce que nous nous s'efforçons d'expliquer (l'explanandum). En outre, quoi que nous pensions comprendre de cette intelligence transcendante, nous le ferons sur la base de notre compréhension préalable de l'intelligence humaine, de sorte que la conjecture $e$ intelligentia rerum paraît condamnée à une inexorable circularité. Comme le formulait Maurice Merleau-Ponty en 1945:

On sait que la pensée classique cherche en somme à expliquer les concordances par un monde en soi ou par un esprit absolu. De telles explications empruntent au phénomène de la rationalité tout ce qu'elles peuvent avoir de convainquant, elles ne l'expliquent donc pas et ne sont jamais plus claires que lui. La Pensée absolue n'est pas plus claire pour moi que mon esprit fini, puisque c'est par lui que je la pense ${ }^{30}$.

Ou bien l'intellect divin n'explique rien dans la mesure exacte où il est incompréhensible, ou bien il n'explique rien parce que nous le comprenons exclusivement par analogie avec notre vie intentionnelle. Par conséquent, le Dieu des philosophes peut-il vraiment être présenté comme un principe ou une source d'intelligibilité ? Ne doit-on pas affirmer que le phénomène de la rationalité constitue plutôt un mystère premier et indépassable, qu'il ne s'agit pas de dissiper en posant une Raison préexistante et transcendante à l'œuvre dans le sensible, mais qu'il revient à la phénoménologie d'élucider de manière immanente? En ce sens, si le professeur Grondin souligne à juste titre que la philosophie moderne a fâcheusement tendance à «scier la branche sur laquelle elle est assise » en prétendant dépasser la métaphysique générale ou l'ontologie, on pourrait regretter que celui-ci rompe avec l'orientation anti-spéculative de la phénoménologie - véritable "programme de recherche" qui était encore celui de Gadamer et de Ricœur et dont nous sommes bien loin d'avoir épuisé toutes les promesses.

\section{Bibliographie}

Comte-Sponville, André. L'esprit de l'athéisme. Introduction à une spiritualité sans Dieu, Éditions Albin Michel, coll. "Le Livre de Poche", 2006.

- Dieu et la science (avec F. Euvé et G. Lecointre), Paris, Presses de l'ENSTA, coll. "Les Tables rondes de l'ENSTA ParisTech", 20 Iо.

Gadamer, Hans-Georg. L'art de comprendre. Écrits II, Herméneutique et champ de l'expérience humaine. Paris, Éditions Aubier Montaigne, I991.

28. "Le positivisme, c'est la science sans métaphysique; le scientisme, c'est la science comme métaphysique. Mieux vaut le positivisme que le scientisme, et mieux vaut la métaphysique que le positivisme! ", André Comte-Sponville, 2010, p. I7-I8.

29. Jean Grondin, 2013, p. I34.

30. Maurice Merleau-Ponty, I945, p. 470. 
Grondin, Jean. Le tournant herméneutique de la phénoménologie, Paris, Presses universitaires de France, coll. "Philosophies", 2003.

- Introduction à la métaphysique, Montréal, Presses de l'Université de Montréal, 2004.

. L'herméneutique, Paris, Presses universitaires de France, coll. «Que saisje?", 2006.

—. "La thèse de l'herméneutique sur l'être ", Revue de métaphysique et de morale, vol. 4, $\mathrm{n}^{\circ}$ 52, 2006, p. 469-48I.

. Du sens des choses. L'idée de la métaphysique, Paris, Presses universitaires de France, coll. de métaphysique Chaire Étienne Gilson, 20I3.

Kant, Emmanuel. Euvres philosophiques, tome I: Des premiers écrits à la Critique de la raison pure (I747-I787), Paris, Éditions Gallimard, La Pléiade, trad. I980.

Merleau-Ponty, Maurice. Phénoménologie de la perception, Paris, Éditions Gallimard, coll. «Tel», I945.

Ricœur, Paul. Du texte à l'action. Essais d'herméneutique II, Paris, Éditions du Seuil, coll. «Points", I 986.

Taylor, Charles. "A Philosopher's Postcript: Engaging the Citadel of Secular Reason ", dans P. J. Griffiths, \& R. Hütter (dir.), Reason and the Reasons of Faith, Londres, T \& T Clark International, 2005, p. 339-353.

. Philosophical Arguments, Cambridge, Harvard University Press, I995.

. "Reply and Re-articulation", dans J. Tully (dir.), Philosophy in an Age of Pluralism: The Philosophy of Charles Taylor in Question, Cambridge University Press, I994, p. 213-257. 